\title{
Comparison of cell repair mechanisms by means of chromosomal aberration induced by proton and gamma irradiation - preliminary results ${ }^{\star}$
}

\author{
A. Kowalska ${ }^{1,2,3}$, K. Czerski ${ }^{1, a}$, M. Kaczmarski ${ }^{1}$, M. Lewocki ${ }^{1,4}$, B. Masojć ${ }^{4}$, and A. Łukowiak $^{1}$ \\ 1 Institute of Physics, University of Szczecin, ul. Wielkopolska 15, 70-451 Szczecin, Poland \\ 2 Maritime University of Szczecin, Wały Jagielońskie 1-2, 70-500 Szczecin, Poland \\ 3 Joint Institute of Nuclear Research, Joliot-Curie 6, 141980 Dubna, Russia \\ 4 Departament of Oncology, Pomeranian Medical University, ul. Strzałowska 22, 71-730 Szczecin, Poland
}

Received 13 January 2014 / Received in final form 17 July 2014

Published online 19 March 2015

(c) The Author(s) 2015. This article is published with open access at Springerlink.com

\begin{abstract}
DNA damage of peripheral blood lymphocytes exposed to gamma and proton irradiation is studied by means of chromosome aberrations to validate the efficiency of the repair mechanisms of individual cells. A new method based on an observed deviation from the Poisson statistics of the chromosome aberration number is applied for estimation of a repair factor $(R F)$ defined as a ratio between originally damaged cells to the amount of finally observed aberrations. The repair factors are evaluated by studying the variance of individual damage factors in a collective of healthy persons at a given dose as well as by using the chi-square analysis for the dose-effect curves. The blood samples from fifteen donors have been irradiated by Co60 gamma rays and from nine persons by $150 \mathrm{MeV}$ protons with different doses up to 2 Gy. A standard extraction of lymphocyte has been used whereby dicentrics, acentrics and rings have been scored under a microscope. The $R F$ values determined for the proton radiation are slightly larger than for gamma rays, indicating that up to $70 \%$ DNA double strand breaks can be repaired.
\end{abstract}

\section{Introduction}

Microscopic events leading to DNA damage by ionizing radiation may occur at different spatial and temporal scales. Considerable progress in modelling of such processes has been made during the last decade [1]. Monte Carlo [2] models together with sophisticated combination of fast electron dynamics and ab initio molecular dynamics [3] showed that it is the first stage after ions or high energy photons enter human tissue, i.e. energy transfer to the electrons on the scale of $10^{-14}$ seconds is responsible not only for further track evolution [4] but also for the biological response to ionizing radiation [5].

Chromosome aberrations in peripheral blood lymphocytes are considered the most sensitive and reliable indicators of genetic damage usually related to the double strand breaks of the DNA in the cell nucleus induced by ionizing radiation. Moreover, the number of chromosome aberrations can be associated to the radiation dose by the so-called dose-effect relationship allowing for a biological

\footnotetext{
* Contribution to the Topical Issue "Nano-scale Insights into Ion-beam Cancer Therapy", edited by Andrey V. Solov'yov, Nigel Mason, Paulo Limão-Vieira and Malgorzata SmialekTelega.

a e-mail: czerski@physik.tu-berlin.de
}

dosimetry $[6,7]$. This relationship is characteristic for different types of ionizing radiation and describes not only its biological effectiveness, providing an important marker for the cancer risk, but also includes information about repair mechanisms of individual cells. They are controlled by different enzymatic processes [8] and could be recently visualized by monitoring the $\gamma$-H2AX foci [9] or by using PFGE method [10].

Even though the repair mechanisms can be very effective, the models of the biological response to ionizing radiation still do not take into account these effects directly. Thus, the statistical methods introduced by Edwards et al. [11] based on looking for a reduced appearance of multiple chromosome aberrations and corresponding deviation from the Poisson distribution remains one of the most powerful tools for studying repair mechanisms. A similar approach can be applied by using the generalized Poisson distribution $[12,13]$. Both methods work very well, however, only for large radiation doses for which the number of observed multiple aberrations can be fitted accurately. For low doses we have recently proposed to use deviations from the Poisson statistics of all determined aberrations by means of the variance analysis of the individual damage factor (IDF) or alternatively by the chi-square analysis of the dose-effect curve [14]. The method provides very robust results estimating the 
number of repaired chromosomes (double strand breaks) larger than $50 \%$ for peripheral blood lymphocytes exposed to the gamma radiation of Co60 at the dose of $2 \mathrm{~Gy}$.

In the present paper, the new method has been applied to the results obtained for blood samples irradiated by unmodulated protons of energy $150 \mathrm{MeV}$. The protons being charged particles produce rather small tracks (of several $\mathrm{nm}$ ) of ionizing events along their paths, while gamma rays ionize the tissue through secondary processes leading to a uniform energy deposition in a cell. In terms of their Linear Energy Transfer (LET), the respective LET value for $150 \mathrm{MeV}$ protons is about $0.570 \mathrm{keV} / \mu \mathrm{m}$ and further increases while protons slow down in the samples. In the case of Co60 gamma radiation the value of LET is estimated at a much lower value of about $0.200 \mathrm{keV} / \mu \mathrm{m}$ [19]. Therefore, we could test whether the differences in the ionization density can influence the efficiency of the repair mechanisms.

In the present paper the new method to the results obtained for irradiation of blood samples by $150 \mathrm{MeV}$ protons is shown. The protons being heavy charged particles produced a rather small radius (of several $\mathrm{nm}$ ) of ionized matter along the ion track, while the gamma rays ionize the tissue by secondary processes leading to a uniform energy deposition in a cell. On the other hand, the $150 \mathrm{MeV}$ protons have the LET value of about $0.570 \mathrm{keV} / \mu \mathrm{m}$ increasing further during the energy loss in samples, much larger than $0.200 \mathrm{keV} / \mu \mathrm{m}$ for the Co60 gamma radiation. Therefore, we could test whether the differences in the ionization density can influence the efficiency of the repair mechanisms.

\section{Materials and methods}

The experiment with Co60 gamma rays was performed at the West Pomeranian Oncology Center and the aberration analysis was done at the Cytogenetics of Pomeranian Medical University in Szczecin, Poland. Proton irradiation and sample analysis was done in Joint Institute of Nuclear Research (JINR) in Dubna, Russia.

In the experiment with gamma rays, a standard facility for the Co60 radiotherapy was used. Protons were accelerated by JINRs synchrophasotron to the original energy of $171 \mathrm{MeV}$. The beam was then defocused and conducted to the energy degraders made of plexiglass (thickness of $40 \mathrm{~mm}$ ) and water (thickness of $12 \mathrm{~mm}$ ) to finally reduce the proton energy down to $150 \mathrm{MeV}$ $(\mathrm{LET}=0.570 \mathrm{keV} / \mu \mathrm{m})$. Doses (up to $2 \mathrm{~Gy})$ and the spatial dose distribution were measured by means of calibrated ionization chambers and compared to theoretical calculations based on the stopping-power dependence and the energy straggling of the beam. The dose rate for proton irradiation was $1 \mathrm{~Gy} / \mathrm{min}$.

Blood samples were obtained from healthy, young volunteers males and females. Ethical approval was obtained for this study and all participants gave informed consent. The number of donors was fifteen in the case of the gamma irradiation and nine for proton irradiation. All blood samples were collected by syringes with sodium

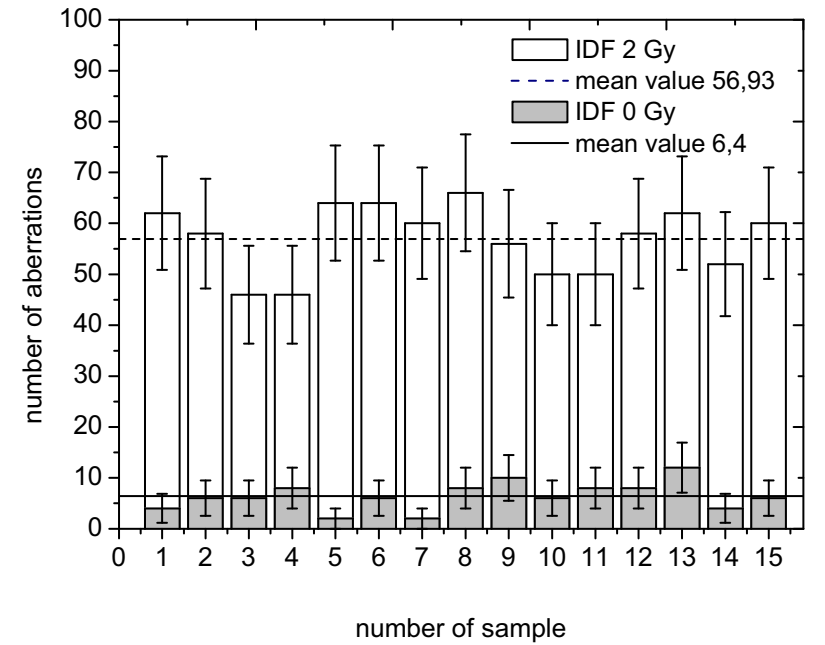

Fig. 1. Number of aberrations induced by gamma irradiaotion (0 Gy and 2 Gy) with the assessed $A D F$ values.

heparin as anticoagulant. Freshly collected blood was diluted in chromosome medium (Gibco) supplemented with PHA-C (Gibco) and then exposed to Co60 gamma rays $(\mathrm{LET}=0.2 \mathrm{keV} / \mu \mathrm{m})$ at the dose rate of $0.5 \mathrm{~Gy} / \mathrm{min}$ for $0.5 \mathrm{~Gy}$ and $1 \mathrm{~Gy} / \mathrm{min}$ for the doses up to $2 \mathrm{~Gy}$. All activities were conducted in room temperature $\left(210^{\circ} \mathrm{C}\right)$. Blood used to the experiment with protons was diluted after irradiation. For all patients at least two samples of diluted blood were prepared. For one of two samples obtained from each patient, the 2 Gy dose was given. The second sample was not exposed to radiation. Irradiated and non-irradiated blood samples were incubated at $37{ }^{\circ} \mathrm{C}$. Cell division was stopped after $48 \mathrm{~h}$ by adding Colchicine. Lymphocytes were isolated from other blood cells by centrifugation, treated with hypotonic solution $(0.56 \% \mathrm{KCl})$ and fixed three times with methanol/glacial acetic acid $(5: 1)$, mixed with the same amount of $0.9 \% \mathrm{NaCl}$.

Harvested lymphocytes were deposited on microscope slides and stained with Giemsa. All slides were coded and analyzed under light microscope. Types of aberrations considered were: dicentrics, rings and acentric fragments.

Summing all aberrations found, the individual damage factor $(I D F)$ can be defined as ratio of metaphases possessing aberrations $(A)$ to all analyzed metaphases $(X)$.

$$
I D F=\frac{A}{X} \times 100 \%
$$

The corresponding average damage factor $(A D F)$ can be calculated as follows:

$$
A D F=\frac{\sum_{i} A_{i}}{\sum_{i} X_{i}} \times 100 \%
$$

The number of observed aberrations presented in Figures $1-3$ is normalized to 100 metaphases and thus matches the $I D F$ value. 
Table 1. Fitted parameters of the dose-effect lines.

\begin{tabular}{ccccc}
\hline & Slope $(a)$ & Uncertainity $(\Delta a)$ & Offset $(b)$ & Uncertainty $(\Delta b)$ \\
\hline Gamma irradiation & 18.6 & 4.2 & 7.1 & 3.7 \\
$150 \mathrm{MeV}$ proton irradiation & 31.1 & 2.7 & 13.9 & 2.4 \\
\hline
\end{tabular}

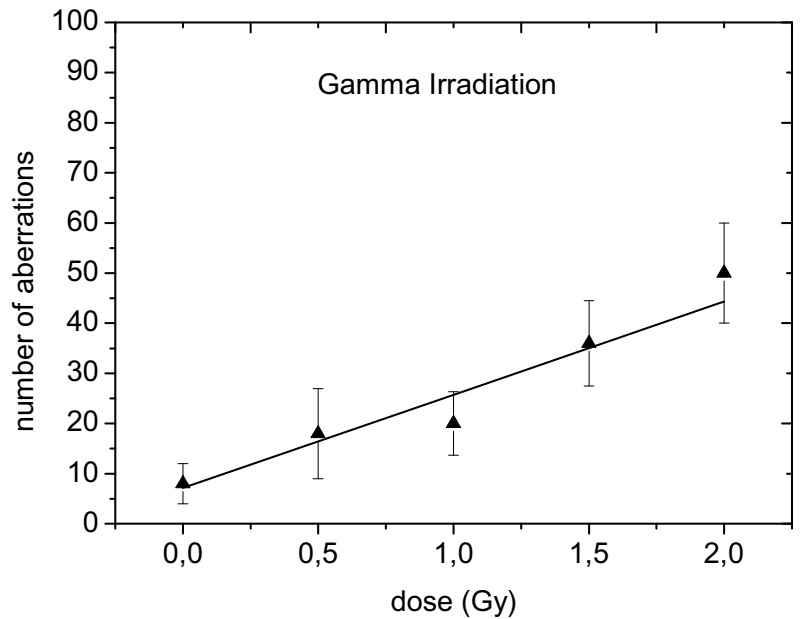

Fig. 2. Dose-response curve for gamma irradiation of the sample number 10 .

\section{Experimental results and statistical analysis}

The IDF values obtained for fifteen blood samples exposed to gamma rays at the dose of 2 Gy are presented in Figure 1 together with the results of non-irradiated samples. Assuming the Poisson distribution, the error bars of the $I D F$ values corresponding to the $68 \%$ confidence level are simply equal to square root of the aberration number $(\sigma P)$. However, as can be seen, error bars of only two points instead of the expected five points do not overlap the $A D F$ value. This implies that the Poisson statistics overestimates the variance of the experimental data around the mean value, which can be independently calculated by the formula:

$$
\sigma_{R}^{2}=\frac{1}{n-1} \sum_{i=1}^{15}\left(I D F_{i}-A D F\right)^{2} .
$$

As discussed in our previous publication [14], overestimation of the variance may be caused by the existence of strong aberration repair mechanisms which reduce the number of originally damaged chromosomes. We suppose that original number of aberrations $A^{\prime}$ that could be observed in absence of any repair mechanism as a result of the original double strand breaks decreases after the time of cell culture $(48 \mathrm{~h})$ and the number of finally observed aberrations amounts to $A$. A repair factor $(R F)$, measuring the strength of repair mechanisms at given radiation dose, can be then defined as the ratio:

$$
R F=\frac{A^{\prime}}{A} \geq 1
$$

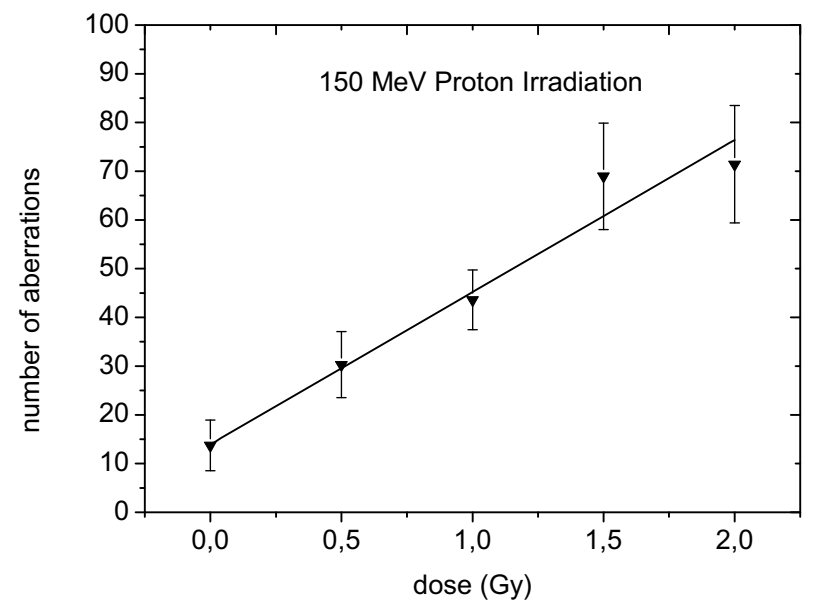

Fig. 3. Dose-response curve for a sample exposed to $150 \mathrm{MeV}$ proton irradiation.

Since the aberration numbers can be expressed by the corresponding Poissons variances, the repair factor can also be calculated as follows:

$$
R F=\frac{\sigma_{P}^{2}}{\sigma_{R}^{2}}
$$

The percentage of repaired cells can then be obtained according to the formula:

$$
R F=\frac{A^{\prime}-A}{A^{\prime}} \times 100 \%=\frac{R F-1}{R F} \times 100 \% .
$$

The $R F$ value determined for the 2 Gy gamma dose amounts to $2.6 \pm 0.3$, e.g. $(61 \pm 4) \%$ of originally damaged cells could be repaired (see Tab. 2). Uncertainties of the above quantities have been estimated assuming normal distribution of the standard deviations, which leads to a $68 \%$ confidence interval given by the chi-square distribution. Homogeneity of the patient group studied was tested applying the $t$-test and creating two subgroups for males ( 8 samples) and females ( 7 samples). The mean values of the subgroups were not significantly different, the corresponding $p$-value amounted to 0.43 . The difference between the variances of the subgroups according to the Levene's test was also not significant, its $p$-value being equal to 0.165 . On the other hand, small differences in the radiosensitivity between various persons are expected. However, these systematic differences are much smaller than Poisson uncertainties of the group studied which is documented by $R F$ larger than unity.

Similar effects can be studied by means of the doseeffect curves. The experimental results obtained for the gamma and proton irradiation are illustrated in Figures 2 and 3, respectively. In both cases the linear dependence is assumed (see Tab. 1). Error bars counted 
Table 2. Comparison of repair factors.

\begin{tabular}{cccc}
\hline & \multicolumn{2}{c}{ Gamma Irradiation } & $150 \mathrm{MeV}$ Proton Irradiation \\
\hline RF & IDF (2 Gy exposition) & $\chi^{2}$ analysis (dose-effect dependence) & $\chi^{2}$ analysis (dose-effect dependence) \\
Repaired & $2.6 \pm 0.3$ & $2.4 \pm 0.3$ & $3.8 \pm 0.8$ \\
aberrations (\%) & $61 \pm 4$ & $58 \pm 5$ & $74 \pm 6$ \\
\hline
\end{tabular}

by Poisson statistics are again overestimated compared to the dispersion of data points around the fitted line.

To compare the distances of the experimental points to the regression line with their standard deviations $\sigma_{i}$, we define the chi-square function

$$
\chi^{2}=\frac{1}{n-2} \sum_{i=1}^{n} \frac{\left(y_{i}-a x_{i}-b\right)^{2}}{\sigma_{i}^{2}} .
$$

The chi-square value should be equal to one when the distances of experimental points to the fitted line with the parameters $a$ and $b$ correspond to the real standard deviations $\sigma_{R_{i}}$

$$
\chi_{R}^{2}=1=\frac{1}{n-2} \sum_{i=1}^{n} \frac{\left(y_{i}-a x_{i}-b\right)^{2}}{\sigma_{R_{i}}^{2}} .
$$

Taking into account Poisson standard deviation $\sigma_{P}$ and assuming that real standard deviations are smaller than the Poissons by a factor $k$, the following expression can be obtained:

$$
\begin{aligned}
\chi_{P}^{2} & =\frac{1}{n-2} \sum_{i=1}^{n} \frac{\left(y_{i}-a x_{i}-b\right)^{2}}{\sigma_{P_{i}}^{2}} \\
& =\frac{1}{n-2} \sum_{i=1}^{n} \frac{\left(y_{i}-a x_{i}-b\right)^{2}}{k^{2} \sigma_{R_{i}}^{2}} \\
& =\frac{1}{k^{2}} \frac{1}{n-2} \sum_{i=1}^{n} \frac{\left(y_{i}-a x_{i}-b\right)^{2}}{\sigma_{R_{i}}^{2}}=\frac{1}{k^{2}} \chi_{R}^{2}=\frac{1}{k^{2}} .
\end{aligned}
$$

Finally, the repair factor is defined in the same way as for the $I D F$ analysis:

$$
R F=\frac{\sigma_{P}^{2}}{\sigma_{R}^{2}}=\frac{\chi_{P}^{2}}{\chi_{R}^{2}}=\chi_{P}^{2}=\frac{1}{k^{2}} .
$$

The $R F$ values for the gamma and proton irradiation based on the chi-square method are presented in Table 2. In the case of gamma irradiation, the chi-square method delivers very similar $R F$ values. On the other hand, $R F$ obtained for protons is slightly larger, possessing, however, a larger uncertainty. The uncertainties of the repair factors result from the uncertainties of parameters of the fitted regression line.

\section{Discussion and conclusion}

A reduction of the variance of the number of chromosome aberration compared to that predicted by the Poisson distribution could be observed for both radiation types and for both analysis methods: IDF and the dose-effect curve. Although, the ionizing densities of $150 \mathrm{MeV}$ protons are much higher than for gamma photons for which we have estimated a larger repair factor, corresponding to about $70 \%$ of repaired aberrations. That also contradicts the larger relative biological effectiveness $(R B E)$ of the proton beam compared to the gamma rays assumed in the radiotherapy to be of about 1.1. Our results are in fair agreement with this value. Taking the ratio of corresponding slopes of the linear dose-effect dependences we obtain the $R B E$ value of $1.67 \pm 0.41$. On the other hand, the repair factor previously determined [14] for a C12 beam of $R B E=2.4$ reached a value of 1.5 only, being much lower than for gamma rays $(R F=2.5)$ and protons $(R F=3.8)$.

It can be argued that the above differences result from the fact that for both protons and carbon ions, the probability for creation of the chromosome aberration does not follow the Poisson but rather the Neuman type. A distribution [15]. The latter represents a product of probabilities for hit a single cell and creation of the chromosome aberration in the cell. Consequently, the expected value of this distribution is also a product of the expected values of the partial Poisson distributions mentioned before. Thus, our method based on the assumption that the chromosome aberrations and their expected value obey the Poisson statistics can also be applied to the proton and heavy ion irradiation experiments. Therefore, we would prefer another explanation for the large repair factor of the proton beam. It has been lately speculated [16] that the energy deposition region of fast protons (radius of the proton ion track) can be much larger than the range of cascade electrons produced due to the ionization process. That could reduce the ionization density for protons and increase the repair probability. A strong candidate for the extension of the interaction radius of protons is an electron wake-field wave [17]. Of course, a separate question is whether the energy stored in the wake-field wave is large enough to lead to chromosome aberrations.

Another effect we could observe is that the repair factor based on the dose-effect curve is comparable to that obtained within the IDF analysis at the dose of 2 Gy. It confirms our assumption in the derivation of the chisquare function that repair factor is not dependent on the dose. On the other hand, there are some indications that the repair mechanisms should work much more effectively at low doses [18], which could be investigated in future studies.

Summarizing, the method for evaluation of the repair factor presented here works also for the proton beam. Its simplicity and applicability for studies on the biological 
response to different ionizing radiation at low irradiation doses makes it very useful for further experiments in this field.

\section{References}

1. N.I. Nakajima et al., PLoS One 8, e70107 (2013)

2. H. Nikjoo, P. O'Neill, D.T. Goodhead, M. Terrissol, Int. J. Radiat. Biol. 71, 467 (1997)

3. E. Surdutovich, A.V. Yakubovich, A. Solovyov, Sci. Rep. 3, 1289 (2013)

4. G. Schiwietz, K. Czerski, M. Roth, F. Staufenbiel, P.L. Grande, Nucl. Instrum. Methods Phys. Res. B 226, 683 (2004)

5. M. Toulemonde, E. Surdutovich, A.V. Solovyov, Phys. Rev. E 80, 031913 (2009)

6. A.C. Upton, Environmental Health Perspectives 52, 31 (1983)

7. Cytogenetic Dosimetry: Applications in Preparedness for and Response to Radiation Emergencies (IAEA, Vienna, 2011), vol. 8, p. 45

8. K. Sakata, M. Someya, Y. Matsumoto, M. Hareymana, Radiat. Med. 25, 433 (2007)

9. L. Mah, C. Orlowski, K. Ververis, R.S. Vasireddy, A. El-Osta, T.C. Karagiannis, Genome Integrity 2:3 (2011)
10. J.T. Dunnen, G.B. Omen, in Protocols in Human Molecular Genetics: Methods in Molecular Biology, edited by C. Mathew (The Humana Press Inc., Clifton, 1992), vol. 9 , p. 313

11. A.A. Edwards, D.C. Lloyd, R.J. Purrott, Radiat. Environ. Biophys. 16, 89 (1979)

12. C.M.S. Lee, F. Famoye, Biometrical J. 38, 299 (1996)

13. K. Janardan, D. Schaeffer, Biometrical J. 19, 599 (2007)

14. A. Kowalska, K. Czerski, M. Kaczmarski, M. Lewocki, B. Masojć, to be published in Rad. Res.

15. E. Gudowska-Nowak, R. Lee, E. Nasonova, S. Ritter, M. Scholz, Adv. Space Res. 39, 249 (2005)

16. E. Surdutovich, A.V. Solov'yov, Phys. Rev. E 82, 051915 (2010)

17. R.H. Ritchie, W. Brandt, P.M. Echenique, Phys. Rev. B 14, 4808 (1976)

18. L.E. Feinendegen, M. Pollycove, C.A. Sondhaus, Nonlinearity Biology, Toxicol. Med. 2, 143 (2004)

Open Access This is an open access article distributed under the terms of the Creative Commons Attribution License (http://creativecommons.org/licenses/by/4.0), which permits unrestricted use, distribution, and reproduction in any medium, provided the original work is properly cited. 